\title{
FINITE-ELEMENT FORMULATIONS FOR PROBLEMS OF LARGE ELASTIC-PLASTIC DEFORMATION
}

\author{
R. M. MCMEEKING and J. R. RICE \\ Division of Engineering, Brown University, Providence, RI 02912, U.S.A.
}

(Received 8 May 1974; revised 19 August 1974)

\begin{abstract}
Ahstract-An Fulerian finite element formulation is presented for problems of large elastic-plastic flow. The method is based on Hill's variational principle for incremental deformations, and is ideally suited to isotropically hardening Prandtl-Reuss materials. Further, the formulation is given in a manner which allows any conventional finite element program, for "small strain" elastic-plastic analysis, to be simply and rigorously adapted to problems involving arbitrary amounts of deformation and arbitrary levels of stress in comparison to plastic deformation moduli. The method is applied to a necking bifurcation analysis of a bar in plane-strain tension.

The paper closes with a unified general formulation of finite element equations, both Lagrangian and Eulerian, for large deformations, with arbitrary choice of the conjugate stress and strain measures. Further, a discussion is given of other proposed formulations for elastic-plastic finite element analysis at large strain, and the inadequacies of some of these are commented upon.
\end{abstract}

\section{INTRODUCTION}

Many elastic-plastic problems of interest involve large deformations and the present paper is concerned with finite element formulations for this class of problem. Such formulations are also relevant to small deformation elastic-plastic problems. Indeed the conventional "small strain" formulation in stress analysis can be inadequate for small strain problems not only when increments of rotation greatly exceed those of strain (buckling of slender members) but also when representative stress levels have attained a magnitude comparable to that of the plastic hardening modulus. The latter can occur after only minute deformations for lightly hardening metals. These situations relate more to the shape of the member under consideration and to intrinsic material properties, rather than to the smallness or largeness of strain per se, but are best approached in a rigorous finite deformation context.

Several workers have proposed and utilized finite element schemes for problems of finite deformation, and these include diverse formulations of both the Lagrangian (material) and Eulerian (spatial) type. The next section gives a brief review of these and includes some comments on ambiguities in certain of the approaches to elastic-plastic behavior. Thereafter we proceed directly to an Eulerian formulation which is ideally suited to the large deformation of Prandtl-Reuss materials. This is given in a manner which allows the straightforward adaptation of any existing small strain program to finite deformation analysis. It also serves as a basis for analysis of the possible inadequacies of the conventional small strain formulation. The program is applied to a numerical study of the initiation of plastic bifurcation in a plane strain tensile bar.

Finally, in the concluding section of the paper we have presented a general formulation of the finite deformation problem in finite-element terms. By appropriate specialization this can be made to coincide with various separate formulations, both Lagrangian and Eulerian, and provides a suitable basis for comparison of the different approaches as well as for a fuller discussion of ambiguities in other elastic-plastic formulations as noted above.

\section{REVIEW OF FINITE ELEMENTS FOR FINITE DEFORMATIONS}

The description Lagrangian is attached to large deformation finite element programs which use a mesh of elements representing some fixed reference state for strain. A scheme of this type 
was proposed by Hibbitt, Marcal and Rice[1], who derive their finite element rate equilibrium equations from the principle of virtual work for large deformation. They identify four stiffness terms, which are named small strain stiffness, initial load stiffness, initial strain stiffness and initial stress stiffness. In elastic-plastic analysis, all of these must be calculated for each increment of deformation and the last three have a complicated form. Needleman [2] has a similar Lagrangian scheme, but he derives his equations from a variational principle due to Hill [3]. A third Lagrangian formulation was presented by Felippa and Sharifi[4], who intend to place no limitation on the size of an increment of deformation. Higher order terms are thus introduced into their stiffness. However, these terms are not significant in a tangent modulus approach to elastic-plastic or non-linear elastic analysis, since the increment of deformation must be small in any case to ensure that the constitutive rate moduli do not change significantly from one increment to the next. For the materials mentioned the method of [4] coincides with that of [1].

The Eulerian formulation which is based on a mesh representing the current state of deformation has been used by Yaghmai and Popov[5]. In their presentations they derive equations from a current configuration version of the variational principle used in [4] and solve an elastic and an elastic-plastic problem. Sharifi and Popov [6] extended the method of [5] to elastic-plastic analysis of infinitesimal strains but finite rotations, although they do not seem to consider the possibility that plastic deformation moduli may be of a size comparable to current stress levels. Another Eulerian formulation is due to Gunasekera and Alexander [7], who base their equations for a Prandtl-Reuss material on the principle of virtual work. The finite element equilibrium equations they obtain are correct if their stress $\sigma$ is interpreted as the 2nd Piola-Kirchoff stress, but a statement to this effect is not made. If their $\sigma$ is so identified, the constitutive law used does not then seem to constitute an adequate form of the Prandtl-Reuss equations. There also seems to be an ambiguity in work on an Eulerian formulation for Prandtl-Reuss materials by Argyris and Chan [8], who do not make a clear definition of their stress and strain terms. Two interpretations appear possible, but neigher constitutes an adequate finite element method. This point is discussed in the final section of the paper, along with further comments on [6] and [7]. Osias [9] has an Eulerian scheme, which admits non-symmetric constitutive laws through a Galerkin method. He derives the same rate equilibrium equations as obtained here, but his constitutive law leads to a non-symmetric stiffness.

\section{AN EULERIAN FINITE ELEMENT FORMULATION FOR FINITE DEFORMATION}

A concise formulation of rate equilibrium at arbitrary amounts of deformation is given by the following form of the virtual work equation, which has been cited by Hill[3].

$$
\int_{V^{\circ}} \dot{t}_{i j} \delta\left(\frac{\partial v_{j}}{\partial X_{i}}\right) \mathrm{d} V^{\circ}=\int_{V^{\circ}} \dot{b}_{\imath} \delta v_{1} \mathrm{~d} V^{\circ}+\int_{S^{\circ}} \dot{f}_{i} \delta v_{1} \mathrm{~d} S^{\circ}
$$

where all integration extents are in the reference configuration and $\mathbf{X}$ is the position vector of a material point in that reference state. $t$ is the non-symmetric nominal stress, which is defined so that the force vector $f$ per unit reference area of a surface having the reference unit normal $n^{\circ}$ is given by $f_{i}=n_{i} t_{i j}$, and $\mathbf{b}$ is the body force per unit reference volume. Rates are indicated by the superposed dot and $\delta \mathbf{v}$ is an arbitrary virtual velocity variation which disappears where velocity rates are prescribed, i.e. on $S^{\circ}-S_{T}^{\circ}$, where $S_{T}^{\circ}$ is the reference surface on which tractions are prescribed. Now, by the device of choosing a reference state that is instantaneously coincident with the current state, $t$ may be simply related to a spin-invariant stress rate, the Jaumann or 
co-rotational rate of Kirchhoff stress $\tau^{*}$, more suited to use in constitutive relations. The relationship is [3]

$$
i_{i j}=\tau_{i j}^{*}-\sigma_{k j} D_{k i}-\sigma_{i k} D_{k j}+\sigma_{i k} v_{j, k}
$$

where $\sigma$ is the Cauchy stress, $v_{i, k}$ is $\partial v_{j} / \partial x_{k}$ where $\mathbf{x}$ is the position vector of a material point in the current state and $\mathbf{D}$ is the rate of deformation tensor such that

$$
D_{i j}=\frac{1}{2}\left(v_{i, j}+v_{\mathrm{j}, i}\right)
$$

The Kirchhoff and Cauchy stresses are related through

$$
\tau_{i j}=J \sigma_{i j}
$$

where $J$ is the ratio of volume in the reference state to volume in the current state (so that $J=1$ instantaneously).

Under these circumstances (1) becomes [3]

$$
\int_{V}\left[\tau_{i j}^{*} \delta D_{i j}-\frac{1}{2} \sigma_{i j} \delta\left(2 D_{i k} D_{k j}-v_{k, i} v_{k, j}\right)\right] \mathrm{d} V=\int_{S} \dot{f}_{i} \delta v_{i} \mathrm{~d} S+\int_{V} \dot{b}_{i} \delta v_{i} \mathrm{~d} V
$$

where all integration extents are in the current configuration. $\dot{\mathbf{f}}$ and $\dot{\mathbf{b}}$ are still nominal force intensity rates, but with respect to the current areas.

The principle (5) may be used to formulate an Eulerian finite element method. For this purpose we define $\{\dot{\psi}\}$, which is the vector of rates of nodal degrees of freedom and $\{v\},\{\dot{\boldsymbol{\epsilon}}\}$ and $\{D\}$, which are respectively the vectors of components of the velocity, the infinitesimal strain rate and the deformation rate all at a point in an element. In a conventional small strain program the position and element-type dependent matrices $[N]$ and $[B]$ relate $\{v\}$ and $\{\dot{\epsilon}\}$ to the rates of degrees of freedom of the undeformed mesh so that

$$
\{v\}=[N]\{\dot{\psi}\}, \quad\{\dot{\boldsymbol{\epsilon}}\}=[B]\{\dot{\psi}\} .
$$

However in an Eulerian program, the same matrices $[N]$ and $[B]$, as used in a conventional small strain program for elements of a given kind, relate $\{v\}$ and $\{D\}$ to the rates of degrees of freedom $\{\dot{\psi}\}$ of a mesh representing the current geometry, i.e.

$$
\{v\}=[N]\{\dot{\psi}\}, \quad\{D\}=[B]\{\dot{\psi}\} .
$$

The matrices are related by

$$
\left[B_{i j}\right]=\frac{1}{2}\left[N_{i}\right]_{, j}+\frac{1}{2}\left[N_{j}\right]_{i,}
$$

where $\left[N_{l}\right],\left[B_{i j}\right]$ are rows of $[N],[B]$ defined so that

$$
v_{i}=\left[N_{i}\right]\{\dot{\psi}\}, \quad D_{i j}=\left[B_{i j}\right]\{\dot{\psi}\} .
$$


The finite element rate equilibrium equations obtained from (5) have two stiffness terms. The first stiffness arises from $\int_{V} \tau_{i j}^{*} \delta D_{i j} \mathrm{~d} V$, and the other is an initial stress stiffness $\left[k_{s}\right]$ arising from the remainder of the left-hand side of (5). Note however that there is no initial strain stiffness. $\left[k_{s}\right]$ is obtained from the relationship

$$
\{\delta \dot{\psi}\}^{T}\left[k_{s}\right]\{\dot{\psi}\}=\int_{V}\left(\delta v_{k, i} \sigma_{i j} v_{k, j}-2 \delta D_{k i} \sigma_{i j} D_{k j}\right) \mathrm{d} V
$$

or

$$
\left[k_{s}\right]=\int_{V}\left(\left[N_{k}\right]_{. i}^{T} \sigma_{i j}\left[N_{k}\right]_{. j}-2\left[B_{k i}\right]^{T} \sigma_{i i}\left[B_{k j}\right]\right) \mathrm{d} V,
$$

where $\{\delta \dot{\psi}\}$ is the variation in nodal rates of the current mesh that generates $\delta \mathbf{v}, \delta \mathbf{D}$. Hence the complete finite element equations are

$$
\left(\int_{V}[B]^{T}[C][B] \mathrm{d} V+\left[k_{s}\right]\right)\{\dot{\psi}\}=\{\dot{P}\}
$$

where

$$
\{\dot{P}\}=\int_{V}[N]^{T}\{\dot{b}\} \mathrm{d} V+\int_{S_{T}}[N]^{T}\{\dot{f}\} \mathrm{d} S
$$

$[C]$ is the rate independent incremental constitutive matrix appearing in the form

$$
\left\{\tau^{*}\right\}=[C]\{D\}
$$

and $\left\{\tau^{*}\right\}$ is a vector of components of $\tau^{*}$. When the prescribed force rates are such that $\{\dot{b}\}$ and $\{\dot{f}\}$ on $S_{T}$ cannot be fully identified, $\{\dot{P}\}$ divides into a prescribed part and a geometric part, linear in $\{\dot{\psi}\}$. The last part must be added as another stiffness term in the manner described by Hibbitt, Marcal and Rice [1].

The form (5) given above and thus this finite element method is of general validity. Although the constitutive law of interest

$$
\tau_{i j}^{*}=\mathscr{L}_{i j k l} D_{k l}
$$

or equivalently $\left\{\tau^{*}\right\}=[C]\{D\}$ is materially objective to superposed spins, the tensor $\mathscr{L}$ in its most general form may be dependent on the total deformation from some prior state including total rotations. However (5) and the associated finite element method is of greatest convenience for problems in strain rate independent isotropically hardening elastic-plastic materials with rate constitutive laws written in terms of $\tau^{*}$ and $D$ and dependent only on material parameters and the state of stress. This class of materials includes those with different loading and unloading paths. For these materials the finite element rate equilibrium equations for the current mesh are independent of the total deformations. Furthermore the first stiffness in this case is formed identically in terms of constitutive rate moduli as in small strain programs. The second stiffness has a simple universal form in terms of $\sigma$. Indeed, the nature of these stiffnesses allows a convenient adaption of small strain programs with an appropriate constitutive law. 


\section{CONSTITUTIVE LAWS}

The constitutive law used to formulate any finite element equations at finite deformation may lead to a non-symmetric stiffness. The most general case leading to a symmetric stiffness is a law derived from Hill's [3] homogeneous quadratic rate potential $\Phi$, so that

$$
i_{i l}=\frac{\partial \Phi}{\partial\left(\partial v_{j} / \partial X_{i}\right)}=\frac{\partial^{2} \Phi}{\partial\left(\partial v_{j} / \partial X_{i}\right) \partial\left(\partial v_{l} / \partial X_{k}\right)} \frac{\partial v_{l}}{\partial X_{k}}
$$

and

$$
\Phi=\frac{1}{2} t_{i}\left(\nabla_{x} v\right) \partial v_{i} / \partial X_{i}
$$

Such laws are symmetric and rate independent and have been phrased in terms of conjugate variables of stress and strain. All elastic constitutive laws with work-potentials and elastic-plastic laws with a normality rule expressed in terms of conjugate variables of stress and strain take this form $[3,10,11]$. The elastic-plastic laws have zero order dependence on strain rate, since the response at yield is governed by the direction of loading. However, when both the elastic and the elastic-plastic moduli are applied, to a strain rate tangential to the yield surface, the same stress rate results. It is then obvious that at yield (13) is still uniquely satisfied and $\Phi$ has continuous first variation with respect to strain rate. For materials for which $\Phi$ exists, (1) may be rewritten as

$$
\delta\left\{\int_{V^{\circ}} \Phi\left(\nabla_{X} v\right) \mathrm{d} V^{\circ}-\int_{V^{\circ}} \dot{b}_{i} v_{i} \mathrm{~d} V^{\circ}-\int_{S ¥} \dot{f}_{i} v_{i} \mathrm{~d} S^{\circ}\right\}=0
$$

and a current configuration version of (14) is derived from (5) so that

$$
\delta\left\{\int_{V} U(\mathbf{D}) \mathrm{d} V-\frac{1}{2} \int_{V} \sigma_{i j}\left(2 D_{i k} D_{k j}-v_{k, i} v_{k, j}\right) \mathrm{d} V-\int_{V} \dot{b}_{i} v_{i} \mathrm{~d} V-\int_{s_{\tau}} \dot{f}_{i} v_{i} \mathrm{~d} S\right\}=0
$$

where

$$
U=\frac{1}{2} \tau_{i j}^{*}(\mathbf{D}) D_{i j}
$$

and

$$
\tau_{i j}^{*}=\frac{\partial U}{\partial D_{i j}}=\frac{\partial^{2} U}{\partial D_{i j} \partial D_{k l}} D_{k l}=\mathscr{L}_{i j k l} D_{k l}
$$

Hill[12] has studied the transformation between constitutive matrices for different stress rate and strain rate measures and has also shown[3] that the existence of $\Phi$ for a material implies the existence of $U$, and of similar quadratic potentials when constitutive laws are phrased in terms of conjugate stress and strain measures; the converse is so also.

\section{PRANDTL-REUSS EQUATIONS}

The classical Prandtl-Reuss equations for isotropically hardening materials, as presented, e.g. by Hill ([13], pp. 15-39), are not restricted to small-strain, although it is necessary to specify for them a suitable materially objective stress rate when principal deformation axes are rotated. The 
Jaumann rate $\sigma^{*}$ of Cauchy stress coincides with $\dot{\boldsymbol{\sigma}}$ in the absence of rotation and hence may be chosen for their generalization. The equations are then

$$
D_{i j}=\frac{1+\nu}{E} \sigma_{i j}^{*}-\frac{\nu}{E} \delta_{i j} \sigma_{k k}^{*}
$$

for elastic loading or any unloading and

$$
D_{i j}=\frac{1+\nu}{E} \sigma_{i j}^{*}-\frac{\nu}{E} \delta_{i j} \sigma_{k k}^{*}+\frac{9 \sigma_{i j}^{f} \sigma_{k l}^{\prime} \sigma_{k l}^{*}}{4 h \bar{\sigma}^{2}}
$$

for loading at yield, where

$$
\begin{aligned}
& \sigma_{i j}^{\prime}=\sigma_{i j}-\frac{1}{3} \delta_{i j} \sigma_{k k,} \\
& \bar{\sigma}^{2}=\frac{3}{2} \sigma_{i j}^{\prime} \sigma_{i j}^{\prime}
\end{aligned}
$$

$\nu$ is Poisson's ratio, $E$ is Young's modulus, $h$ is the slope of the Cauchy stress-logarithmic plastic strain curve for a simple tension test and $\delta_{i j}$ is the Kronecker delta. The equation (18) may be inverted to show that for plastic loading

$$
\sigma_{i j}^{*_{j}}=\frac{E}{1+\nu}\left[\delta_{i k} \delta_{j l}+\frac{\nu}{1-2 \nu} \delta_{i j} \delta_{k l}-\frac{3 \sigma_{i j}^{\prime} \sigma_{k l}^{\prime}\left(\frac{E}{1+\nu}\right)}{2 \vec{\sigma}^{2}\left(\frac{2}{3} h+\frac{E}{1+\nu}\right)}\right] D_{k l}
$$

There is no rate potential for this law, and when Osias used it as a generalization of the Prandtl-Reuss equations he obtained a non-symmetric stiffness. Hibbitt et al. used the same generalization, and Budiansky [14] has proposed laws which can be shown to be equivalent to the equations $(18,19)$ given above $[15,16]$. Budiansky's laws, which use Green strain and which are referred to curvilinear co-ordinates, were used by Needleman [2] and Chen[17]. A generalization of the Prandtl-Reuss equations, which does admit the potential $U$ and does lead to symmetric stiffness is

$$
\tau_{i j}^{*}=\frac{E}{1+\nu}\left[\delta_{i k} \delta_{l l}+\frac{\nu}{1-2 \nu} \delta_{i j} \delta_{k l}-\frac{3 \sigma_{i}^{\prime} \sigma_{k l}^{\prime}\left(\frac{E}{1+\nu}\right)}{2 \bar{\sigma}^{2}\left(\frac{2}{3} h+\frac{E}{1+\nu}\right)}\right] D_{k l}
$$

for plastic loading and

$$
\tau_{i j}^{*}=\frac{E}{1+\nu}\left[\delta_{i k} \delta_{j l}+\frac{\nu}{1-2 \nu} \delta_{i j} \delta_{k l}\right] D_{k l}
$$

for elastic loading or any unloading. This law is referred to a current configuration and is equivalent to (17-19) to within terms of stress divided by elastic modulus since volume change is taken to be purely elastic. Needleman and Chen note this equivalency and although they postulate that Budiansky's equations govern material response, they use equations equivalent to 
(20) for their calculations. This last form has also been introduced and discussed by Hutchinson [16]. It should be noted that the elasticity in (20) is approximately derivable from a work potential to within terms of stress divided by elastic modulus in comparison to unity [18]. As stated previously a rate potential must evidently exist for elastic response, and (20) but not (19) is in accord with this. The matrix $[C]$ for the law (20) is homogeneous of degree zero in $\{\dot{\psi}\}$, in the usual manner, for elastic-plastic materials at yield. Since this $[C]$ is also the constitutive matrix relating stress rate to $\{\dot{\epsilon}\}$ in a small strain program, the stiffness of such a program calculated in current configuration constitutes one term of the stiffness of the Eulerian finite deformation program. To this must be added $\left[k_{s}\right]$ to form the complete stiffness.

\section{CONVENTIONAL SMALL-STRAIN FORMULATION}

The conventional small-strain principle of virtual work may be written as

$$
\int_{V} \sigma_{i j} \dot{\epsilon}_{i j}^{\dagger} \mathrm{d} V=\int_{V} b_{i} v_{i} \dagger \mathrm{d} V+\int_{S} f_{i} v_{i} \dagger \mathrm{d} S
$$

where $\sigma$ is the stress field in equilibrium with surface tractions $\mathbf{f}$ and body forces $\mathbf{b}, \dot{\boldsymbol{\epsilon}}^{+}$is the virtual rate of the infinitesimal strain tensor and is compatible with the virtual velocity field $\mathbf{v}^{\dagger}, S$ is the surface of the body and $V$ is the volume of the body. All these functions are associated with the undeformed state and the difference between deformed and undeformed volume is neglected. In that case, since the virtual field $\mathbf{v}^{\dagger}$ may be taken as time independent and also taken as some arbitrary variation $\delta \mathrm{v}$, vanishing wherever $\mathrm{v}$ is prescribed on $S$ (i.e. on $S-S_{\mathrm{T}}$ ),

$$
\int_{V} \dot{\sigma}_{i j} \delta \dot{\epsilon}_{i j} \mathrm{~d} V=\int_{V} \dot{b}_{i} \delta v_{i} \mathrm{~d} V+\int_{S} \dot{f}_{i} \delta v_{i} \mathrm{~d} S
$$

Suppose that the constitutive rate law has the form

$$
\dot{\sigma}_{i j}=\mathscr{L}_{i j k l} \dot{\epsilon}_{k l}
$$

where $\mathscr{L}=\mathscr{L}(\boldsymbol{\sigma})$ is symmetric in interchange of $i \mathrm{j}, \mathrm{kl} . \mathscr{L}$ takes different forms for plastic loading vs unloading, but $\dot{\boldsymbol{\sigma}}$ varies continuously with $\dot{\boldsymbol{\epsilon}}$. Then (22) is equivalent to[19]

$$
\begin{gathered}
\delta I[\mathbf{v}]=0, \quad \text { where } \\
I[\mathbf{v}]=\int_{V} \frac{1}{2} \dot{\sigma}_{i j}(\dot{\boldsymbol{\epsilon}}, \boldsymbol{\sigma}) \dot{\epsilon}_{i j} \mathrm{~d} V-\int_{V} \dot{b}_{i} v_{i} \mathrm{~d} V-\int_{S^{T}} \dot{f}_{i} v_{\mathrm{i}} \mathrm{d} S
\end{gathered}
$$

and this variational principle may serve as the basis for a finite element formulation. Alternatively and equivalently, (22) could serve as well and would be needed for non-symmetric $\mathscr{L}$. Using the previously defined terms $\{\dot{\psi}\},\{v\},\{\dot{\epsilon}\},[N]$ and $[B]$ of (6) for small strain analysis, and rendering $I$ of (24) stationary for the discrete class of fields generated by $\{\dot{\psi}\}$, leads to finite element equations in the usual way.

In the introduction certain inadequacies of conventional small strain analysis were mentioned. These may be illustrated by considering equation (15) and assuming now that the current deformed state is indistinguishable from the undeformed state. In this case all stress measures are identical, but all stress rate measures are not. These differ by terms of order $\sigma$ times $\partial \mathbf{v} / \partial \mathbf{x}$. It 
seems plausible to think of $\dot{\sigma}$ in the small strain formulation as being $\tau^{*}$, since this may be assumed to satisfy the postulated Prandtl-Reuss law. Under these circumstances (15) becomes

$$
\delta\left\{\int_{V} \frac{1}{2} \dot{\sigma}_{i j}\left(\dot{\epsilon}_{i j}\right) \dot{\epsilon}_{i j} \mathrm{~d} V-\frac{1}{2} \int_{V} \sigma_{i j}\left(2 \dot{\epsilon}_{i k} \dot{\epsilon}_{k_{j}}-\frac{\partial v_{k}}{\partial X_{i}} \frac{\partial v_{k}}{\partial X_{j}}\right) \mathrm{d} V-\int_{V} \dot{b}_{i} v_{i} \mathrm{~d} V-\int_{S_{T}} \dot{f}_{i} v_{i} \mathrm{~d} S\right\}=0
$$

The additional terms proportional to $\boldsymbol{\sigma}$ that are neglected in a small-strain formulation [compare (24) and (25)] are well-known to be important for slender structural members, in which rates of rotation can greatly exceed rates of straining.

However, even when buckling is not a possibility, it is evident that the neglected terms of the small-strain formulation are of the same importance as those of order $h \dot{\epsilon}^{2}$ arising from the $\dot{\sigma} \dot{\epsilon}$ part of (25), whenever $h$ has a magnitude comparable to that of current stress levels. In this circumstance, predictions of, say, the slope of the overall load-displacement curve in the plastic range cannot be considered accurate. This does not invalidate the solutions from small-strain programs, but means only that one should not consider that the actual stress-strain relation in the hardening range has been precisely modelled. Indeed, the approximation is of the same kind as when the ideally plastic idealization is adopted, with the yield stress taken as some representative flow stress for the amount of deformation at hand. Of course, $h$ is often far greater than current stress levels and then, in the absence of buckling tendencies, small-strain programs are unobjectionable.

\section{SOME NUMERICAL RESULTS}

The Eulerian elastic-plastic finite deformation scheme was incorporated in the manner described into an existing small-strain program, that is a modified version of the relevant part of the MARC program developed by Professor P. V. Marcal, and in use on the IBM 360-67 computer at Brown University. This program models elastic-plastic Prandtl-Reuss materials in a manner described by Tracey[20]. An iterative procedure attempts convergence to a best solution during an elastic-plastic increment, incremental stiffness being calculated by the partial stiffness approach of Marcal and King[21], subsequently modified by Rice and Tracey [22].

A first example illustrates the treatment of finite rotations by this scheme, and uses 4 elements forming a square, which was stressed to represent a state of elastic plane strain. Of the 10 degrees of freedom, 8 were constrained to cause a rotation of $\pi / 4$ radians in first 50 and then 25 increments. The results correspond closely to the exact solution as given by a simple Mohr circle transformation, and are shown in Table 1.

A solution for the initiation of tensile necking of an elastic-plastic bar in plane strain with a constant hardening modulus was also obtained. Osias [9] studied a similar problem. The bar had a ratio of length to width of 3 and the center $\frac{1}{6}$ th of the length was thinned by 0.5 per cent so the analysis could be formulated as a standard deformation problem rather than an eigenvalue

Table 1. Finite rotation by $45^{\circ}$ in the $x-y$ plane for a stressed plane-strain specimen. Comparison of 50 and 25 increment finite element solutions with exact Mohr-circle transformation

\begin{tabular}{lccc}
\hline & $\sigma_{x \mathrm{x}}$ & $\sigma_{\mathrm{yy}}$ & $\sigma_{\mathrm{xy}}$ \\
\hline Before rotation & 1.000 & 0 & 0 \\
Mohr circle & 0.500 & 0.500 & 0.500 \\
50 increments & 0.500 & 0.500 & 0.513 \\
25 increments & 0.501 & 0.499 & 0.526 \\
\hline
\end{tabular}


problem for abrupt bifurcation. One quarter of the bar was analyzed using finite elements and this section is marked OABC in Fig. 1 of the specimen with the thinning greatly exaggerated. There were 432 elements in the mesh and 241 nodes, as shown in Fig. 2. Uniform strain elements in the crossed array shown were chosen because Nagtegaal, Parks and Rice [23] have recently shown this array to be exceptionally free of artificial constraints for incompressible or near incompressible deformation. The material modelled had a ratio of Young's modulus to equivalent Cauchy yield stress of $7.5 \times 10^{2}$, Poisson's ratio was 0.3 and the ratio of hardening modulus to yield stress was constant at 1.25 . The results are shown by the full line in Fig. 3, where the amplitude $\delta$ of the neck (i.e. the width reduction at the center section), normalized by the initial length $L$, is plotted against engineering strain $\gamma=\Delta L / L$. A Fourier analysis of the neck showed that the lateral bar surfaces deformed most dominantly in cosine shape that is predicted from the rigid plastic analysis of Cowper and Onat [24]. In Fig. 3, point $a$ is maximum load, point $b$ is the point at which elastic unloading first occurs and point $c$ corresponds to the specimen illustrated in Fig. 4 with the boundary between the elastic elements and the plastic elements marked $z z$. Those elements, which have unloaded elastically, are above the line $z z$.

It is known [25] that abrupt plastic bifurcation in an elastic-plastic or rigid-plastic body must occur with the overall deformation increasing at a definite initial rate with respect to the bifurcation amplitude. This rate just causes neutral local stress alteration at a single point or locus of points, with subsequent local unloading toward an elastic or rigid state. The dashed line in Fig. 3 is drawn at a slope in accord with that of the initial strain vs bifurcation amplitude slope predicted from the Cowper-Onat solution. The line is located so as to cross the vertical axis at the critical strain predicted from their results (phrased in terms of a critical ratio of hardening rate to true stress, dependent on the current length to width ratio for the bar). It is seen that the large growth of the initial imperfection as predicted from the finite element solution is in close accord with their results. Cowper and Onat found that neutral loading occurs at a point corresponding to

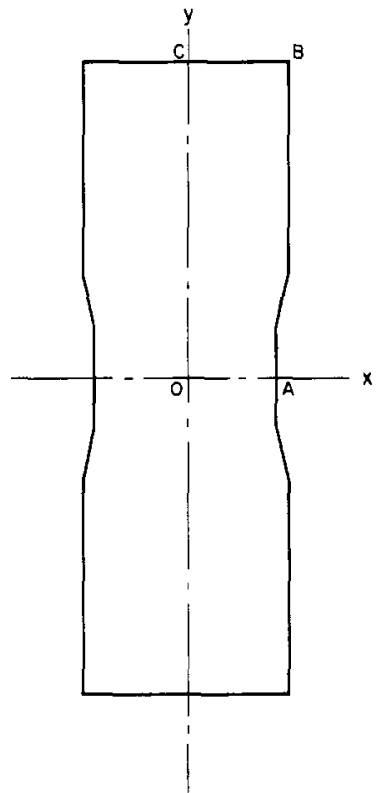

Fig. 1. Specimen for necking analysis, with imperfection shown greatly exaggerated. The actual thinning is 0.5 per cent of full width. 


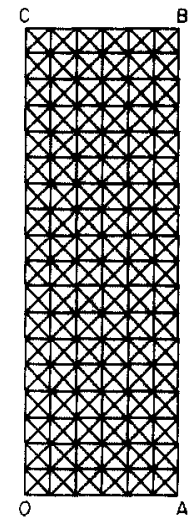

Fig. 2. Finite element mesh for necking analysis.

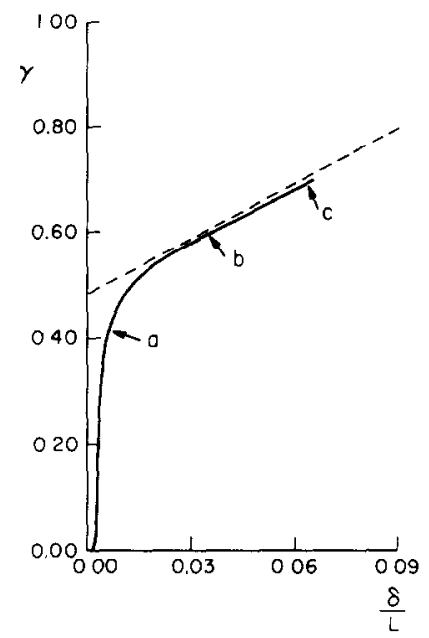

Fig. 3. Engineering strain $\gamma$ vs amplitude of the neck $\delta$ normalized by the undeformed length $L$.

point $C$ in Fig. 1, and suggested that unloading would spread from there towards the side $A B$ of the bar and down to the center line $O A$. This occurred in the numerical analysis of the elastic-plastic bar, and it can be seen from Fig. 3 that once elastic unloading occurred, this analysis began to deviate from dashed line initial slope for the rigid-plastic bar.

The numerical results do not, however, show the inception of unloading at strains very near the amplitude at which large growth of the imperfection sets in, as would be expected from the general result noted earlier for abrupt plastic bifurcations. Also while it is clear that concentration of deformation in the neck and elastic unloading at the end of the bar are both occurring in the numerical analysis, neither are occurring to a great extent. Another aspect of the analysis is that the elements in the neck are elongated in one direction, which has reduced their accuracy. Any further extension of the bar would only aggravate this problem, and thus it would not be profitable to continue the analysis. The elongation of these elements may seem to reduce the value of this Eulerian formulation as opposed to a Lagrangian formulation, where the elements retain their shape. However, the same effect is embedded in the stiffness of the Lagrangian elements and they have no advantage in this respect. 


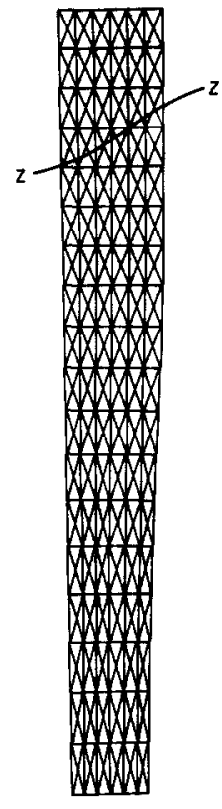

Fig. 4. Deformed mesh at engineering strain 0.69. Elements above line $z z$ have unloaded elastically.

The latter inadequacy at large deformation in the neck is clearly traceable to element size; and smaller elements may also cause the inception of netural loading to take place earlier. For example, Osias [9] used a finer mesh and, while no comparison of his results with the Cowper-Onat solution was made, he did find the inception of unloading at a lower strain and a faster spread of the unloaded zone down the bar axis.

\section{FINITE ELEMENT FORMULATIONS FOR ARBITRARY DEFORMATIONS MAGNITUDES}

Let $\mathbf{e}$ denote any material strain tensor. This is defined on a coordinate system $\mathbf{X}$ in the adopted reference state such that the principal directions of $e$ coincide with the fibers of principal stretch from that state, and so that its principal values $e_{I}, e_{I I}, e_{I I}$ are related to the corresponding stretch ratios $\lambda_{I}, \lambda_{I}, \lambda_{I I}$ by a monotonically increasing function $g(\lambda)$ :

$$
e=g(\lambda), \quad \text { with } \quad g(1)=0 \quad \text { and } \quad g^{\prime}(1)=1 \text {. }
$$

Any strain measure so defined accords with the infinitesimal strain $\epsilon$ at small displacement gradients, and $\dot{\mathbf{e}}=\mathbf{D}$ initially. The most commonly used of this type is Green strain

$$
e^{G}=\frac{1}{2}\left(\lambda^{2}-1\right), \quad e_{i j}^{G}=\frac{1}{2}\left(u_{i, j}+u_{j, 1}+u_{k, i} u_{k, j}\right)
$$

where $\mathbf{u}=\mathbf{x}-\mathbf{X}$ is the displacement from the reference state and now $u_{i, j}=\partial u_{i} / \partial X_{\mathrm{J}}$. We shall also have occasion to use logarithmic strain

$$
e^{L}=\log \lambda
$$


although this cannot be simply expressed in terms of $\partial \mathbf{u} / \partial \mathbf{X}$. However, by series expansion of an arbitrary measure $e$ in terms of $e^{G}$, one obtains

$$
e=e^{G}+(m-1)\left(e^{G}\right)^{2}+\cdots, \quad \text { where } \quad m=\frac{1}{2}\left[g^{\prime \prime}(1)+1\right]
$$

the parameter $m=1$ when $e=e^{G}$, and $m=0$ when $e=e^{L}$. From this an arbitrary strain is given to second order in $\partial \mathbf{u} / \partial \mathbf{X}$ by

$$
e_{i j}=\epsilon_{i j}+\frac{1}{2} u_{k, i} u_{k, j}+(m-1) \epsilon_{i k} \epsilon_{k j}+\cdots, \quad \text { where } \quad \epsilon_{i j}=\frac{1}{2}\left(u_{i, j}+u_{j, l}\right)
$$

Following Hill[11], we define a corresponding family of symmetric conjugate stress measures $\mathbf{s}$, such that $s_{i j} \delta e_{i j}$ is the stress working per unit volume of reference state for arbitrary virtual deformations $\delta$ e. For example the second Piola-Kirchhoff stress $s^{P K}$ is the conjugate to $e^{G}$, where

$$
s_{i j}^{P K}=J \frac{\partial X_{l}}{\partial x_{k}} \sigma_{k l} \frac{\partial X_{j}}{\partial x_{l}} ; \quad J=\operatorname{det}\left(\frac{\partial \mathbf{x}}{\partial \mathbf{X}}\right)
$$

As suitable starting points for incremental finite element formulations, either Lagrangian or Eulerian, one may proceed directly from the rate equilibrium form (1) and transform $t$ to the rate measure most convenient for the constitutive description as done earlier or, alternatively, proceed in the manner of [1] from a virtual work statement in conjugate deformation variables (taken as $\mathrm{e}^{\mathcal{G}}$ and $\mathrm{s}^{\mathrm{PX}}$ in [1]). The end results are the same, and to illustrate this we now take the latter approach, starting from

$$
\int_{V^{\circ}}\{\delta e\}^{T}\{s\} \mathrm{d} V^{\circ}=\int_{V^{\circ}}\{\delta u\}^{T}\{b\} \mathrm{d} V^{\circ}+\int_{S^{\circ}}\{\delta u\}^{T}\{f\} \mathrm{d} S^{\circ}
$$

where $\delta \mathbf{u}$ and $\delta \mathbf{e}$ are associated virtual variations, the forces are nominal, and $\mathbf{s}$, e are any conjugate measures. Matrices $[N]$ and $[B]$ generate the $\delta$ quantities from variations $\{\delta \psi\}$ in the nodal degrees of freedom vector:

$$
\{\delta u\}=[N]\{\delta \psi\}, \quad\{\delta e\}=[B]\{\delta \psi\}
$$

Moreover, $[\boldsymbol{B}]$ is now a function of $\partial \mathbf{u} / \partial \mathbf{X}$, which is different for each different strain measure, although all coincide with a common $\left[B^{\circ}\right]$, the same as that in $(6-8)$, when the current and reference states coincide. Thus the finite element equilibrium equations are

$$
\int_{V^{\circ}}[B]^{T}\{s\} \mathrm{d} V^{\circ}=\{P\}, \quad\{P\}=\int_{V^{\circ}}[N]^{T}\{b\} \mathrm{d} V^{\circ}+\int_{S \uparrow}[N]^{T}\{f\} \mathrm{d} S^{\circ}
$$

and the incremental form is

$$
\int_{V^{\circ}}\left([B]^{T}\{\dot{s}\}+[\dot{B}]^{T}\{s\}\right) d V^{\circ}=\{\dot{P}\}
$$

where $[\dot{B}]^{T}$ will be a linear measure dependent function of $\{\dot{\psi}\}$, and where $\{\dot{P}\}$ is given by (10), 
except for being evaluated in $V^{\circ}$ and on $S^{\circ}$, at least in the normal case as considered here for which the interpolation function $[N]$ has no dependence on $\{\psi\}$.

A more precise identification of $[B]$ and $[\dot{B}]$ is obtained by first noting from (32) that a given column of $[B]$ can be identified as $\partial\{e\} / \partial \psi_{\alpha}$ where $\psi_{\alpha}$ is some member of $\{\psi\}$. Thus if we regard $\{e\}$ as a function of $\partial \mathrm{u} / \partial \mathrm{X}$, and recall the row vectors $\left[N_{i}\right]$ composing $[N]$,

$$
[B]=\frac{\partial\{e\}}{\partial u_{i, j}}\left[N_{i}\right]_{j}
$$

This allows $[\dot{B}]$ to be written as

$$
[\dot{B}]=\{\dot{\psi}\}^{T}\left[N_{k}\right]_{, i}^{T} \frac{\partial^{2}\{e\}}{\partial u_{k, j} \partial u_{i, j}}\left[N_{i}\right]_{,}
$$

The finite element equilibrium equations that follow from (34) are thus

$$
\left(\left[k_{c}\right]+\left[k_{s}\right]\right)\{\dot{\psi}\}=\{\dot{P}\}
$$

where the incremental stiffness arising from the constitutive matrix $[C]$ in

$$
\{\dot{s}\}=[C]\{\dot{e}\}
$$

is

$$
\left[k_{c}\right]=\int_{V^{\circ}}[B]^{T}[C][B] \mathrm{d} V^{\circ}=\int_{V^{\circ}}\left[N_{i}\right]_{, j}^{T} \frac{\partial\{e\}^{T}}{\partial u_{l, j}}[C] \frac{\partial\{e\}}{\partial u_{k, l}}\left[N_{k}\right]_{, l} \mathrm{~d} V^{\circ}
$$

and that arising from the existing stress state (i.e. the initial stress stiffness) is

$$
\left[k_{s}\right]=\int_{V^{\circ}}\left[N_{i}\right]_{, j}^{T} \frac{\partial^{2}\{e\}^{T}}{\partial u_{i, j} \partial u_{k, 1}}\{s\}\left[N_{k}\right], d \mathrm{~d} V^{0}
$$

Both of these are measure dependent, although in a compensatory way, because $[C]$ and the derivatives of $\{e\}$ are measure dependent.

When the adopted measures are Green strain $\left\{e^{G}\right\}$ and second Piola-Kirchhoff stress $\left\{s^{P K}\right\}$, one finds from $(26,32)$ or $(35)$ that a given row $\left[B_{i j}\right]$ of $[B]$, corresponding to the variation $\delta e_{i j}$, is

$$
\left[B_{i j}\right]=\frac{1}{2}\left[N_{i}\right]_{, j}+\frac{1}{2}\left[N_{i}\right]_{, i}+\{\psi\}^{T}\left[N_{k}\right]_{. i}^{T}\left[N_{k}\right]_{j,}
$$

and further the initial stress stiffness is

$$
\left[k_{s}\right]=\int_{V^{\circ}}\left[N_{k}\right]_{, i}^{T} s_{i j}^{P K}\left[N_{k}\right]_{, j} \mathrm{~d} V^{\circ}
$$

This seems to be the most suitable basis for a Lagrangian method, since few other strain measures are readily computed at finite strain. It is important, however, that the constitutive matrix $[C]$ be properly identified. For example, if the Prandtl-Reuss relations are taken to hold in 
terms of $\left\{\tau^{*}\right\}$ or $\left\{\sigma^{*}\right\}$ and $\{D\}$, then the matrix $\left[C^{\circ}\right]$ in

$$
\left\{\tau^{*}\right\}=\left[C^{\circ}\right]\{D\} \quad \text { or } \quad\left\{\sigma^{*}\right\}=\left[C^{\circ}\right]\{D\}
$$

must be properly transformed to [C], as for example in [1].

By contrast, Eulerian finite element formulations are readily made for any choice of measure by taking the reference state to coincide instantaneously with the current state. Then $\{s\}=\{\sigma\}$ and $\{\dot{e}\}=\{D\}$ momentarily and $[B]$ has the small deformation from

$$
\left[B_{i j}\right]=\frac{1}{2}\left[N_{i}\right]_{, j}+\frac{1}{2}\left[N_{\mathrm{J}}\right]_{,,}
$$

when expressed in terms of the current geometry of the element. By setting $\{s\}=\{\sigma\}$ in (40) and evaluating the mixed derivatives of $\{e\}$ from (29) when $\partial u / \partial x=0$, the initial stress stiffness is

$$
\left[k_{s}\right]=\int_{V}\left(\left[N_{k}\right]_{, i}^{T} \sigma_{i j}\left[N_{k}\right]_{, j}+2(m-1)\left[B_{k i}\right]^{T} \sigma_{i j}\left[B_{j k}\right]\right) \mathrm{d} V
$$

Although the stress measures $\{s\}$ and $\{\sigma\}$ coincide, their rates do not and once more special attention must be paid to the matrix $[C]$ in the stiffness

$$
\left[k_{c}\right]=\int_{V}[B]^{T}[C][B] \mathrm{d} V .
$$

The importance of proper identification of $[C]$ in both the Lagrangian and the Eulerian approaches is clearly indicated when the reference state is taken to coincide instantaneously with the current state. Then as Hill[11] has shown

$$
\dot{s}_{i j}=\tau_{i j}^{*}-m\left(\sigma_{i k} D_{k j}+D_{i k} \sigma_{k j}\right),
$$

where $m$ is still the measure dependent coefficient of (28). Since $m=0$ when the adopted measure is logarithmic strain, this means that the Jaumann (co-rotational) rate $\tau^{*}$ of Kirchhoff stress coincides instantaneously with the rate of the stress measure conjugate to logarithmic strain.

Thus, suppose that $\mathscr{L}^{\circ}$ is the constitutive tensor relating $\tau^{*}$ to $\mathbf{D}$. Then the constitutive tensor for any other choice of measure when the current and reference states coincide is given from (46):

$$
\tau^{*}=\mathscr{L}_{i j k l}^{\circ} D_{k l} \quad \text { implies } \quad \dot{s}_{i j}=\left(\mathscr{L}_{i j k l}^{\circ}-m \sigma_{i k} \delta_{j l}-m \sigma_{j k} \delta_{i l}\right) \dot{e}_{k l .}
$$

In the special case $m=0,\left[k_{s}\right]$ and $\left[k_{c}\right]$ of (44) and (45) are then the stiffnesses given earlier in equations $(9,10)$.

Equation (47) without $m$ specifically identified shows the error that would be entailed in writing the Prandtl-Reuss equations in the form (20), but using some arbitrary measure $\dot{s}$ rather than $\tau^{*}$ as the stress rate. This step becomes permissible as an approximation only when the smallest components of $\mathscr{L}$, having the order $h$, greatly exceed components of $\sigma$, the magnitude of which may be represented by $\sigma$. In particular the use of $\dot{s}^{P K}(m=1)$ would imply in uniaxial 
tension or compression a plastic hardening rate of $h+2 \sigma$ when phrased in terms of true stress vs logarithmic strain. If $\sigma$ is of order $h$ this is in conflict with the approximately symmetric tensile and compressive response of initially isotropic materials (when phrased in true stress and logarithmic strain) which the equations are intended to represent [13]. This is pivotal to an assessment of Refs. [6-8]. In the first two the constitutive law (20) is formulated with $\dot{\mathbf{s}}^{\mathrm{PK}}$ taken as the stress rate, although in [7] a definition or description of the adopted measure of stress is not given. However, the terms $[\dot{B}]$ and $\left[k_{s}\right]$ in [7] are clearly computed for Green strain, which requires that the stress rate used must be the rate of Piola-Kirchhoff stress for validity of the equilibrium equations. The problem concerning the constitutive representation is not severe for [6], its intent being only to treat small strains. In that case $h$ often far exceeds the absolute value of $\sigma$ and the imprecision as to stress rate is unimportant. However the adopted constitutive representation is not suitable in [7], which is intended for large plastic deformations, when the absolute value of $\sigma$ can generally be greater than $h$.

Argyris and Chan [8] do not define what measures of either stress or strain are intended in their work. They cannot be inferred unambiguously since no explicit formulae are given for their term analogous to $[\dot{B}]$. Indeed it is not clear that their stress measure is a member of the conjugate family, but for whatever measure it is, the Prandtl-Reuss equations are written with its ordinary time derivative as the stress rate. If their form for $[\dot{B}]$ is intended as for Green strain, then their procedure is identical to that of [7] and all comments concerning [7] also apply to [8]. On the other hand if their stress is meant to be Cauchy or Kirchhoff stress, then the use of its ordinary rather than co-rotational time rate in the Prandtl-Reuss equations creates a constitutive law that is not spin invariant.

Acknowledgements-This study was supported by the National Aeronautics and Space Agency under Grant NGL-40-002-080. We are grateful to Prof. P. V. Marcal for provision of the MARC program and to Mr. D. M. Parks for assistance with its modification along the lines outlined.

\section{REFERENCES}

1. H. D. Hibbitt, P. V. Marcal and J. R. Rice, A finite element formulation for problems of large strain and large displacement. Int. J. Solids Struct. 6, 1069 (1970).

2. A. Needleman, A numerical study of necking in circular cylindrical bars. J. Mech. Phys. Solids 20, 111 (1972).

3. R. Hill, Some basic principles in the mechanics of solids without a natural time, J. Mech. Phys. Solids 7, 209 (1959).

4. C. A. Felippa and P. Sharifi, Computer implementation of nonlinear finite element analysis. in Numerical Solution of Nonlinear Structural Problems (Edited by R. F. Hartung), p. 31. ASME, New York (1973).

5. S. Yaghmai and E. P. Popov, Incremental analysis of large deflections of shells of revolution. Int. J. Solids Struct. 7, 1375 (1971); see also S. Yaghmai and E. P. Popov, Large deflection elastic-plastic analysis of shells of revolution. in First International Conference on Structural Mechanics in Reactor Technology $4 \mathrm{~J}$ (Edited by T. A. Jaegar). Bundesanstalt für Materialprüfung, Berlin (1971).

6. P. Sharifi and E. P. Popov, Nonlinear finite element analysis of sandwich shells of revolution. AIAA Joumal 11, 715 (1973).

7. J. S. Gunasekera and J. M. Alexander, Matrix analysis of the large deformation of an elastic-plastic axially symmetric continuum. in Symposium on Foundations of Plasticity (Edited by A. Sawczuk). p. 125. Noordhoff, Leyden (1973).

8. J. H. Argyris and A. S. L. Chan, Static and dynamic elasto-plastic analysis by the method of finite elements in space and time. in Symposium on Foundations of Plasticity (Edited by A. Sawczuk), p. 147. Noordhoff, Leyden (1973).

9. J. R. Osias, Finite Deformation of Elasto-Plastic Solids, The Example of Necking in Flat Tensile Bars. Ph.D. Thesis, Carnegie-Mellon University, Pittsburgh (1972).

10. R. Hill and J. R. Rice, Elastic potentials and the structure of inelastic constitutive laws. SIAM J. Appl. Math. 25, 448 (1973).

11. R. Hill, On constitutive inequalities for simple materials I and II. J. Mech. Phys. Solids 16, 229-242, 315 (1968).

12. R. Hill, On the classical constitutive relations for elastic/plastic solids in Recent Progress in Applied Mechanics (The Folke Odquist Volume) (Edited by B. Broberg et al.), p. 241. Wiley, New York (1967).

13. R. Hill, The Mathematical Theory of Plasticity, Oxford University Press, London (1950).

14. B. Budiansky, Private communication with W. H. Chen. Ref. 12 in Chen[17] and referenced by Needleman[2].

15. J. R. Rice, Private communication with J. W. Hutchinson (1973). 
16. J. W. Hutchinson, Finite strain analysis of elastic-plastic solids and structures. in: Numerical Solution of Nonlinear Structural Problems (Edited by R. F. Hartung), p. 17. ASME, New York (1973).

17. W. H. Chen, Necking of a bar. Int. J. Solids Struct. 7, 685 (1971).

18. R. M. McMeeking, An Eulerian Finite Element Formulation for Problems of Large Displacement Gradients. S.M. Thesis, Brown University, Providence (1974).

19. D. C. Drucker, Variational principles in the mathematical theory of plasticity. Proc. Symp. Appl. Math. 8, 7 (1958).

20. D. M. Tracey, On the Fracture Mechanics Analysis of Elastic-Plastic Materials Using the Finite Element Method. Ph.D. Thesis, Brown University, Providence (1973).

21. P. V. Marcal and I. P. King, Elastic-plastic analysis of two-dimensional stress systems by the finite element method. Int. J. Mech. Sci. 9, 143 (1967).

22. J. R. Rice and D. M. Tracey, Computational fracture mechanics. in Numerical and Computer Methods in Structural Mechanics (Edited by S. J. Fenves et al.), p. 585. Academic Press, New York (1973).

23. J. C. Nagtegaal, D. M. Parks and J. R. Rice, On numerically accurate finite element solutions in the fully plastic range. Comp. Meth. Appl. Mech. Engng, 4, 153 (1974).

24. G. R. Cowper and E. T. Onat, The initiation of necking and buckling in plane plastic flow. in Proc. Fourth U.S. Cong. Appl. Mech. p. 1023. ASME, New York (1962).

25. J. W. Hutchinson, Post-bifurcation behavior in the plastic range. J. Mech. Phys. Solids 21, 163 (1973). 